Check for updates

Cite this: RSC Adv., 2018, 8, 6452

\title{
Development of a bio-electrospray system for cell and non-viral gene delivery
}

\author{
Myung Chul Lee, $\dagger^{\mathrm{a}}$ Hoon Seonwoo, $\dagger^{\mathrm{b}}$ Pankaj Garg, ${ }^{\mathrm{c}}$ Kyoung Je Jang, ${ }^{a}$

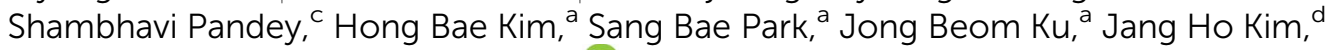 \\ Ki Taek Lim ${ }^{\mathrm{e}}$ and Jong Hoon Chung (D)*ac
}

\begin{abstract}
Bio-electrospray technology is a very attractive tool for preparing scaffolds and depositing desired solutions on various targets by electric force. In this study, we focused on the application of a bio-electrospray (BES) technique to spray cells on the target and to simultaneously deliver genetic constructs into the cells, called non-viral gene delivery-based bio-electrospray (NVG-BES). Using this method, we tried to harvest the electric charge produced during electrospray for the cellular internalization of cationic polymer/DNA nanoparticles as well as the delivery of living cells on the desired substrate. Furthermore, we optimized the voltage, culture medium and polymeric cationic charges for high transfection efficiency and cell viability during NVG-BES. As a result, the solutions used during the NVG-BES process played an important role in improving transfection efficiency. We determined that a voltage of $10 \mathrm{kV}$ with PBS as the spraying solution showed high transfection efficiency, probably due to the facilitation of cationic polymer/DNA nanocomplexes in cellular internalization and their subsequent expression. In conclusion, NVG-BES, as a novel method, is expected to deliver genes to cells and simultaneously deliver transfected cells to any substrate or scaffold.
\end{abstract}

Received 15th November 2017

Accepted 23rd January 2018

DOI: 10.1039/c7ra12477e

rsc.li/rsc-advances non-viral vectors, including synthetic polymers such as polyethylenimine (PEI), poly(L-lysine) (PLL), polyamidoamine (PAMAM) dendrimers and poly(2-dimethyl amino ethyl)methacrylate (PDMAEMA). ${ }^{3,4}$ Several groups also tried to apply mechanical factors as substitutes for viral and non-viral cationic polymer-based gene delivery methods. ${ }^{1}$ Among physical methods such as direct injection, electroporation, and ultrasound-mediated transfection, electroporation is a typical transfection technique that introduces DNA or drugs into cells by increasing the permeability of the cell membranes using electrical fields. ${ }^{5}$ Based on these methods, a new technique for transfection called electrospray (ES) was developed by Okubo et al. $^{6}$ This team showed that the impact by an electrosprayed droplet can be applied to enhance transfection. In the electrospray method, the efficiency of transfection to attached cells was influenced by the charge densities of plasmid-containing sprayed droplets. The size of the droplets could also be an important factor in determining transfection efficiency (Ikemoto et al.). ${ }^{7}$ However, the major limitation of electrospray is its inapplicability to non-adhesive or floating cells. Thus, a more convenient method for delivering genes (regardless of cell type) should be developed.

Bio-electrospray (BES) is a technique to deliver cells by the electrospray method on specific targets. ${ }^{8}$ Although some researchers have proposed risks for BES, Sahoo et al. clearly demonstrated that the viability of bone-marrow derived mesenchymal progenitor/stem cells (BMSC) was not affected by 
BES. ${ }^{8}$ Hall et al. evaluated the genetic safety of BES on human cells. Bio-electrosprayed (BESed) cells showed minor damage relative to the negative control (BES without the application of voltage). ${ }^{9}$ In addition, Eliot et al. reported a transfection method using BES. ${ }^{10}$ However, they used a viral vector as the gene carrier. Viral vector-based gene delivery has potential risks for cells, such as infection or immunogenicity. Thus, we hypothesized that BES with a non-viral vector not only improves transfection efficiency by electric force, but also delivers the cells safely. Herein, we developed a novel method to increase the transfection efficiency and to deliver cells, called non-viral gene delivery-based bio-electrospray (NVG-BES) (Fig. 1). Branched
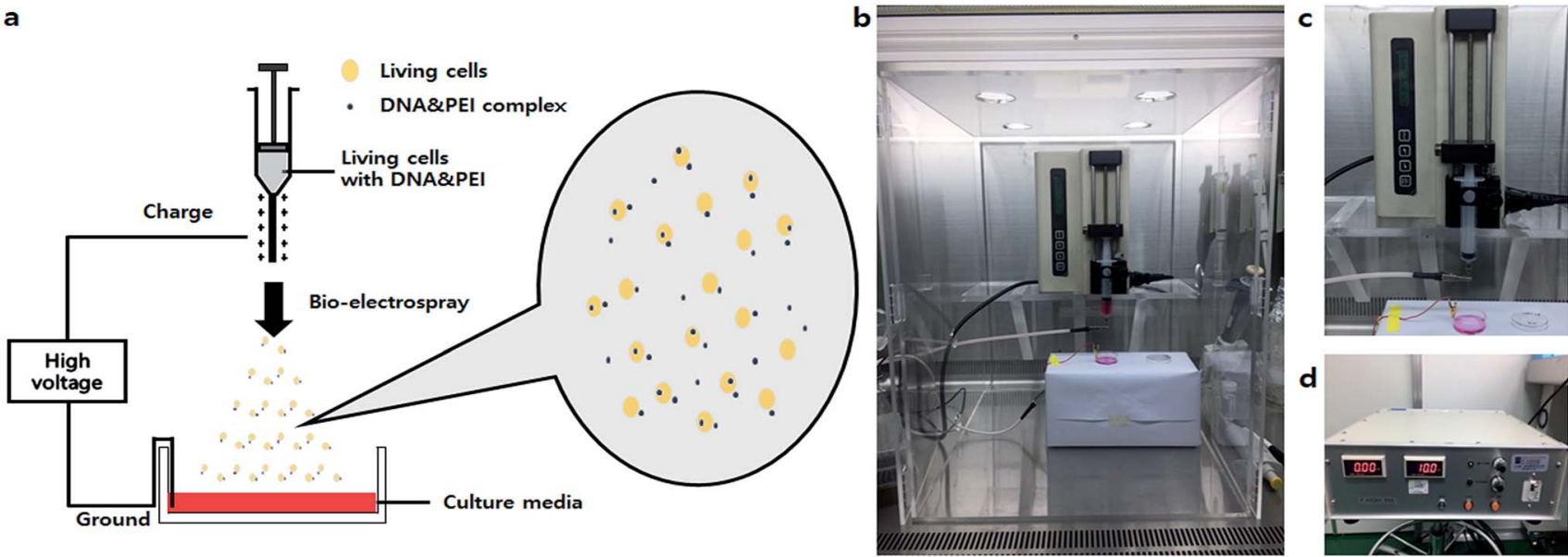

Fig. 1 Non-viral gene delivery-based bio-electrospray (NVG-BES) system. (a) Schematic diagram of the NVG-BES system. The NVG-BES system facilitated introduction of DNA to cells and simultaneously delivered cells to a target. In this method, a cationic polymer was used as non-viral carrier with electric force in a bio-electrospray (BES) system to electrospray living cells onto a target. (b) NVG-BES system on a clean bench. (c) The syringe pump. (d) The high voltage generator.
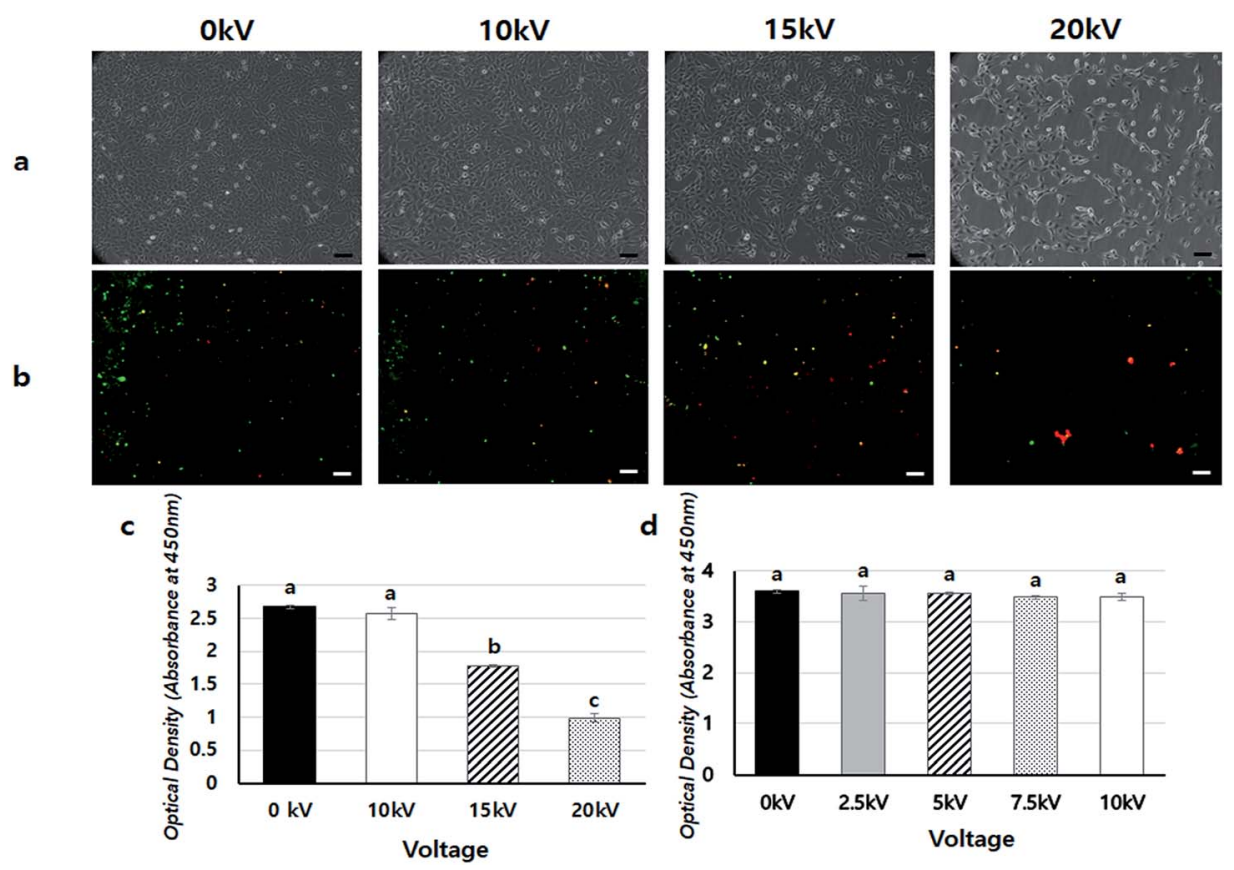

Fig. 2 Cell viability assay: (a) cell morphology 1 day after BES at 0 kV, $10 \mathrm{kV}, 15 \mathrm{kV}$, and $20 \mathrm{kV}$. (b) The results of the live/dead assay 1 day after bioelectrospray at $0 \mathrm{kV}, 10 \mathrm{kV}, 15 \mathrm{kV}$, and $20 \mathrm{kV}$ (green: live cells, red: dead cells). The case of $0 \mathrm{kV}$ is the negative control (no BES). The pictures were taken at $10 \times$ magnification. (scale bars $=100 \mu \mathrm{m}$ ), (c) WST assay of cultured cells 1 day after BES from $0 \mathrm{kV}$ to $20 \mathrm{kV}$. The case of $0 \mathrm{kV}$ is the negative control (no BES) ( $n=3, p<0.05$. Columns with different letters are significantly different according to the Duncan test). (d) WST assay of cultured cells 1 day after BES from $0 \mathrm{kV}$ to $10 \mathrm{kV}$. The case of $0 \mathrm{kV}$ is the negative control (no BES). ( $n=3, p<0.05$. Columns with different letters are significantly different, according to the Duncan test). The results of cell viability assay after BES showed that cells were fine under conditions at $10 \mathrm{kV}$ of BES. However, cell viability was rapidly decreased above $10 \mathrm{kV}$ of BES. 
$25 \mathrm{kD}$ polyethyleneimine (PEI), a well-known cationic polymer used in gene delivery, was used in this study to aid gene transfection due to its well-known properties, such as high availability and high DNA complexation ability. ${ }^{11,12}$ NIH-3T3, a murine fibroblast cell line, was also used in this study. We tried to find the optimal NVG-BES conditions for enhancing the transfection efficiency by checking cell viability after NVG-BES.

\section{Results and discussion}

To confirm the safety of BES, we first investigated the cell viability of $\mathrm{BES}$ at various voltages $(0,10,15$, and $20 \mathrm{kV})$ without PEI. Fig. 2a shows the cell morphology 1 day after BES. All groups successfully deposited cells on tissue culture-polystyrene (TCPS). The cell densities at 0 and $10 \mathrm{kV}$ appeared high, but the cell density at $15 \mathrm{kV}$ was lower than that at $10 \mathrm{kV}$. The cells sprayed at $20 \mathrm{kV}$ had severely low population, which indicates that the cell morphologies appeared fine at $10 \mathrm{kV}$, while above $10 \mathrm{kV}$ cells

a

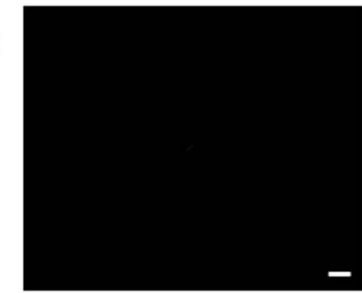

c
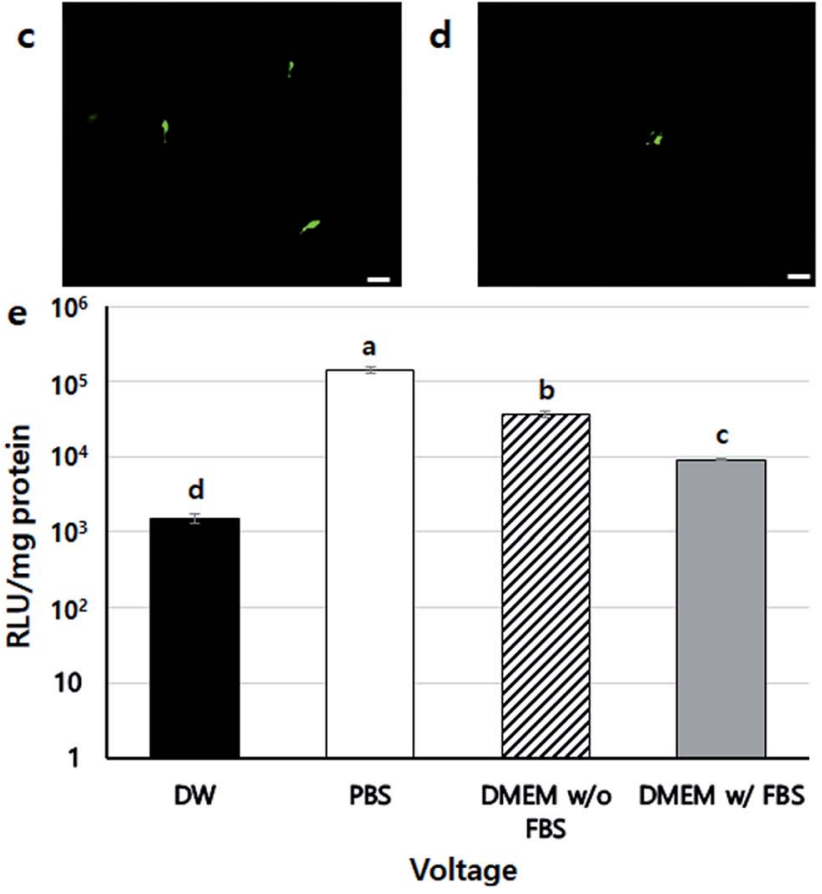

Fig. 3 DNA expression results using NVG-BES with several solutions (a)-(d) GFP expression after NVG-BES transfection with several solutions: (a) DW, (b) PBS, (c) DMEM (w/o FBS), (d) DMEM (w/FBS). GFP expression pictures were taken at $10 \times$ magnification (scale bars $=100$ $\mu \mathrm{m})$. (e) Luciferase activity after BES with several solutions $(n=3 p<$ 0.05. Columns with different letters are significantly different, according to the Duncan-test). Other groups were significantly different to the DW group ( $p<0.001$ ). The use of PBS as BES solution showed higher expression of GFP than the other solutions. appeared damaged and their adhesion decreased. The live/dead assay also showed a similar result (Fig. 2b). The number of red spots (dead cells) appearing on the BESed cells increased with on increasing the applied voltage. Next, we quantitatively measured cell viability using a WST-1 assay (Fig. 2c and d). The viability of sprayed cells decreased remarkably at high voltages (15 and $20 \mathrm{kV}$ groups). However, cell viabilities in the $10 \mathrm{kV}$ group were a

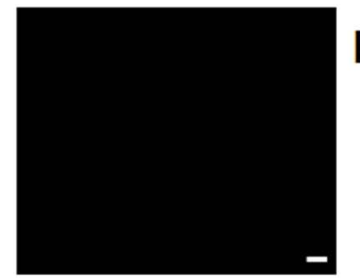

c

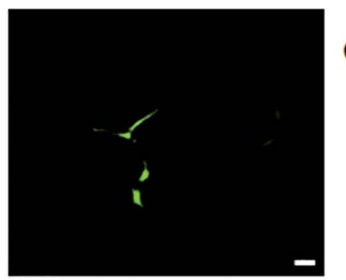

e

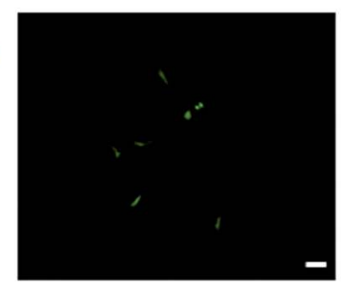

f

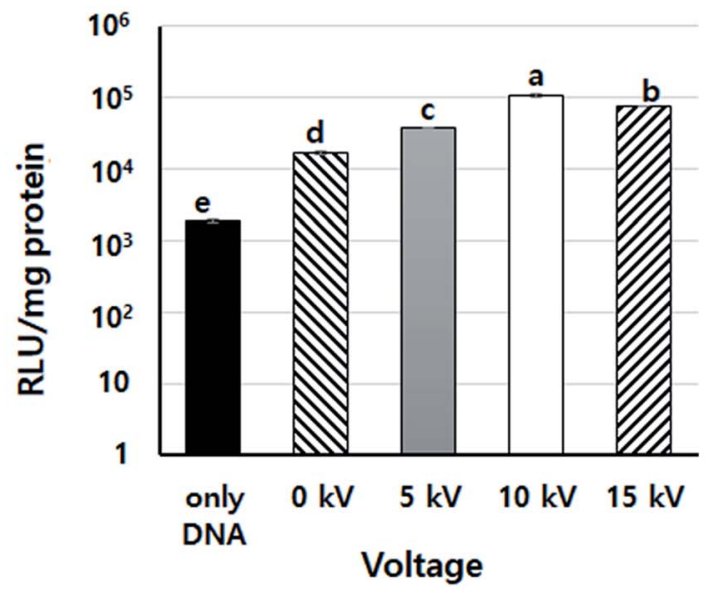

Fig. 4 DNA expression results using NVG-BES at various voltages (a)(f) GFP expression results after bio-electrospray at different voltages: (a) only DNA, (b) $0 \mathrm{kV}$, (c) $5 \mathrm{kV}$, (d) $10 \mathrm{kV}$ and (e) $15 \mathrm{kV}$. The $0 \mathrm{kV}$ group received only $\mathrm{PEI}+\mathrm{DNA}$ (no BES), and the case labelled only DNA received only DNA. GFP expression pictures were taken at $10 \times$ magnification (scale bars $=100 \mu \mathrm{m}$ ). At $10 \mathrm{kV}$ of BES, GFP was expressed more frequently than other groups. (f) Luciferase assay results after BES at different voltages $(n=3, p<0.05$. Columns with different letters are significantly different according to the Duncan test). Other groups were significantly different $(p<0.001)$ than the only DNA group (N.C). The groups using PEI and those applying BES had increased expression of luciferase. In particular, BES at $10 \mathrm{kV}$ had the highest expression level. 
not significantly different from those at $0 \mathrm{kV}$. For more detailed analysis, we repeatedly carried out WST assays of cells BESed from 0 to $10 \mathrm{kV}(0,2.5,5,7.5$, and $10 \mathrm{kV})$. There was no significant difference between the groups, indicating that values below $10 \mathrm{kV}$ are applicable to BES.

Next, the gene transfection ability of NVG-BES was investigated. To verify whether NVG-BES increased the transfection efficiency with or without using a polymer carrier, optimal NVGBES conditions were investigated. First, we tested several solutions such as phosphate buffered saline (PBS), deionised water (DW), DMEM without foetal bovine serum (DMEM w/o FBS) and DMEM with FBS (DMEM w/FBS) to determine the appropriate solution for NVG-BES. The transfection efficiency was verified via green fluorescence protein (GFP) and luciferase assays. The NVG-BES was performed at $10 \mathrm{kV}$ because there was no significant difference in cell viability below $10 \mathrm{kV}$. The PBS group showed better green fluorescence during the GFP assay than the other groups, while the DW group showed very low expression of GFP (Fig. 3a-d). The luciferase assay also showed that the PBS, DMEM w/FBS, and DMEM w/o FBS groups exhibited significantly higher transfection levels $(p<0.001)$ than the DW group. Furthermore, the PBS group exhibited the highest luciferase expression level (Fig. 3e). The GFP and luciferase assays both showed that the best solution for NVG-BES was PBS. The NVG-BES results were affected by the type of solution, that is, the solution used is an important factor in NVG-BES. The DMEM w/FBS group was anticipated to produce lower transfection efficiency than the DMEM w/o FBS group because serum usually interferes with binding between DNA and polymer. ${ }^{13-15}$ As expected, the DMEM w/o FBS group exhibited significantly higher luciferase gene expression than the DMEM w/FBS group.
In addition, the DW group showed the lowest luciferase gene expression. Because cells cannot bear the osmotic pressure when resuspended in DW after harvesting, the cell viability decreased rapidly, resulting in hard gene transfection. Unlike DW, PBS contributes to cell viability as a buffer, helping to maintain cell conditions. Chesnoy et al. suggested that PBS helps stabilize DNA against degradation. ${ }^{16}$ PBS was also used by Roos et al. as a DNA vehicle solution for electroporation and by Kang et al. as a buffer for nucleofection (an application of electrophoresis). ${ }^{17,18}$ Thus, PBS was the best solution and displayed the highest transfection efficiency for NVG-BES.

To find the appropriate voltage, cells with DNA/PEI complex were BESed at various voltages $(0,5,10$, and $15 \mathrm{kV})$. From the GFP expression results, the $10 \mathrm{kV}$ group had more greenfluoresced cells than the other groups (Fig. 4a-e). In the luciferase assay, compared to the only DNA group, all groups showed significantly higher transfection levels $(p<0.001)$ (Fig. 4f). In particular, the gene expression of the $10 \mathrm{kV}$ group was significantly higher than those of the other groups, and the luciferase activity of the $10 \mathrm{kV}$ group was approximately 6 times higher than that of the $0 \mathrm{kV}$ group. Although the gene transfection level of the $15 \mathrm{kV}$ group was also high, cell viability of the $15 \mathrm{kV}$ group decreased severely. The gene transfection level of the $15 \mathrm{kV}$ group was expected to decrease due to cell death induced by excessively high voltage. However, an appropriate voltage may increase the transfection efficiency of NVG-BES. As the cell viability of the $10 \mathrm{kV}$ group was not severely lower than that of the $15 \mathrm{kV}$ group, the appropriate voltage for NVG-BES for transfection was fixed at $10 \mathrm{kV}$.

Fig. 5 shows a comparison of cell viabilities after NVG-BES with only DNA and with DNA/PEI at various voltages $(0,5,10$,

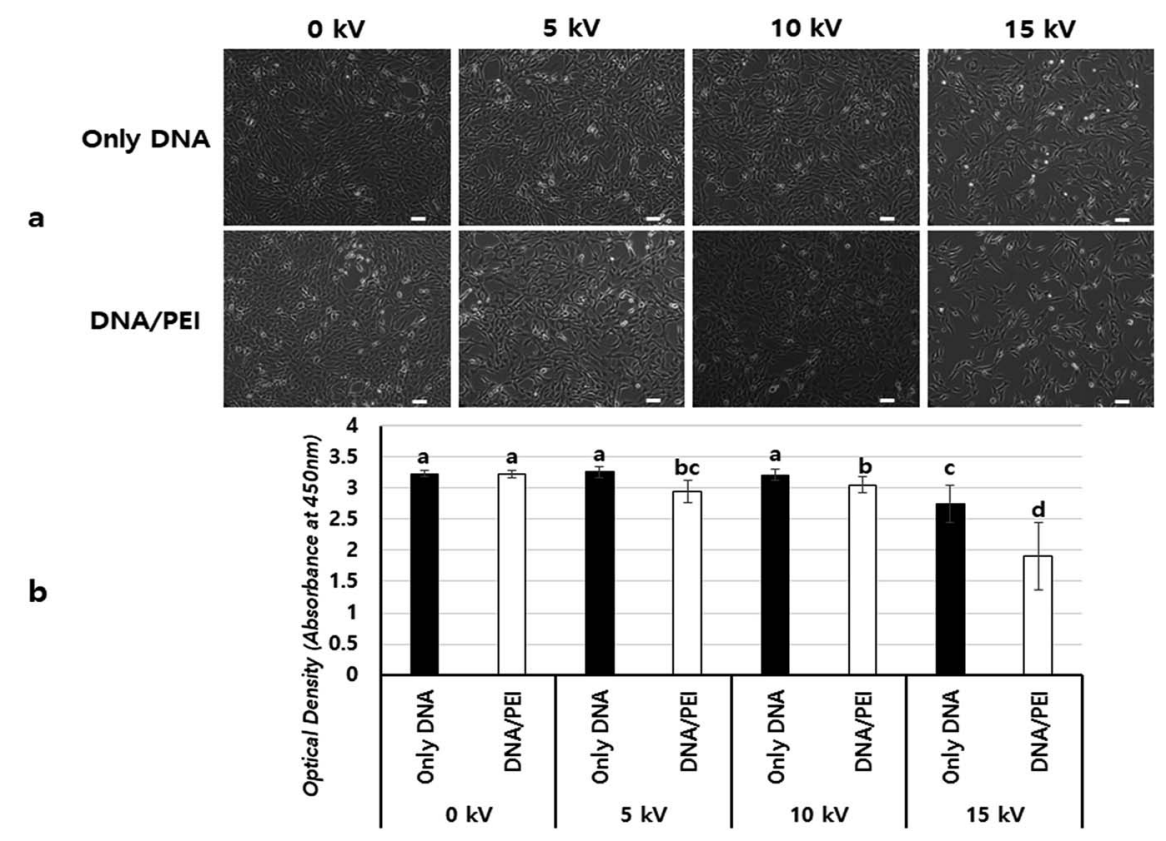

Fig. 5 Comparison of viability between BES with only DNA and BES with PEI \& DNA (a) image of BESed cells after one day at $0 \mathrm{kV}, 5 \mathrm{kV}, 10 \mathrm{kV}$ and $15 \mathrm{kV}$. The pictures were taken at $10 \times$ magnification (scale bars $=100 \mu \mathrm{m})$. (b) WST-1 results $(n=7, p<0.05$. Columns with different letters are significantly different, according to the Duncan test). The case of $0 \mathrm{kV}$ is no BES. However, cell morphology showed no difference at $10 \mathrm{kV}$ of BES, and cell viability of BES with PEI decreased significantly relative to groups of BES without PEI. 
and $15 \mathrm{kV}$ ). Although there were no noticeable morphological differences between the cases with PEI and without PEI (Fig. 4a), the results of the WST-1 assay showed that cell viability in the DNA/PEI group was significantly lower in comparison with the only DNA group when voltages were induced (Fig. 4b). Interestingly, the cell viability of the voltage-induced DNA/PEI group decreased even though there was no significant difference in the cell viability of the only DNA group. It is expected that the change in electric force induced by NVG-BES stimulates the delivery of DNA/PEI complexes into cells, resulting in cytotoxicity. For the relevant application of NVG-BES, finding the appropriate voltage introduced DNA and also minimized the cytotoxicity. Fig. 6 shows proposed NVG-BES mechanisms. Electric shocks were reported to enhance the permeability of the cell membrane, resulting in electroporation., ${ }^{519-21}$ When the electrospray generates electric fields, ${ }^{22,23}$ similar to the electroporation method, the electric fields generated between the needle and culture dish are anticipated to increase cell a

$0 \mathrm{kV}$

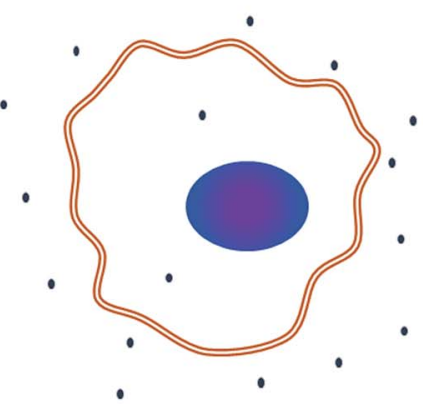

- Normal cellular uptake b

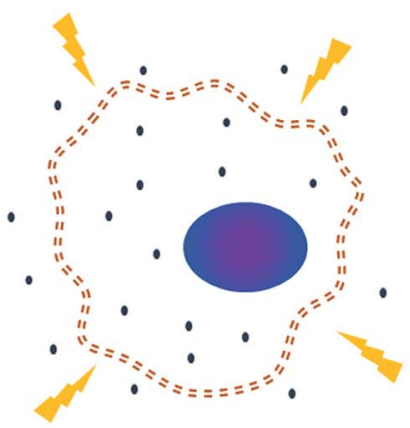

- Cell membrane permeability

- Cellular uptake

- Transfection c

$15 \mathrm{kV}$

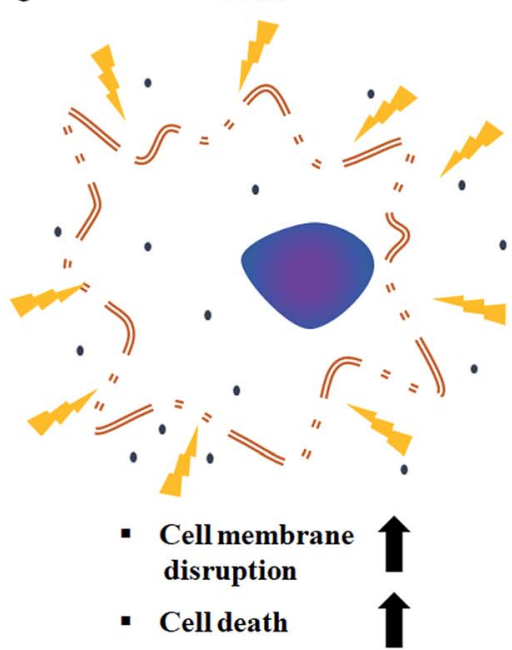

- DNA\&PEI complex

Fig. 6 Proposed NVG-BES mechanism. (a) At 0 kV, normal cellular uptake occurs. (b) At $10 \mathrm{kV}$, cell membrane permeability and cellular uptake are increased by induction of electric field. As a consequence, transfection efficiency increases. (c) Above $10 \mathrm{kV}$, cell membrane disruption and cell death happen due to excessive electric field.
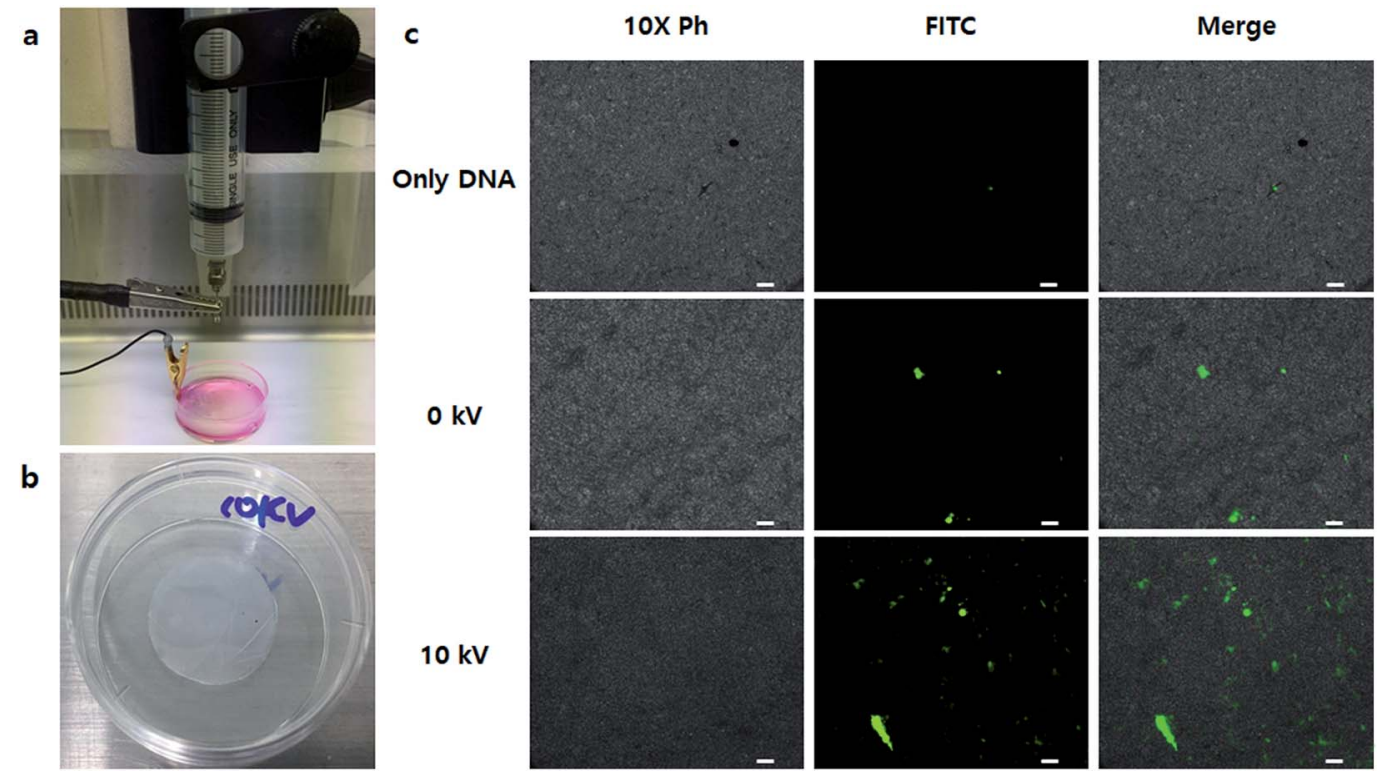

Fig. 7 Delivery transfected cells on the scaffold using the BES transfection method. (a) Bio-electrospray on a PCL nanofiber scaffold. (b) The PCL nanofiber scaffold. (c) The results of delivering transfected cells on a PCL nanofiber scaffold using BES. GFP expression pictures were taken at $10 \times$ magnification (scale bars $=100 \mu \mathrm{m}$ ). It showed that BES transfected living cells and delivered them to a nanofiber scaffold at the same time. 
permeability, leading to increased cellular uptake and transfection efficiency. To reveal the underlying mechanism thoroughly, more studies are needed.

Finally, we applied the NVG-BES to nanofibrous scaffolds to induce simultaneous cell delivery and gene transfection. After nanofibrous scaffolds prepared by the electrospinning method were deposited on a $35 \mathrm{~mm}$ dish, NIH-3T3 cells were BESed onto the nanofibrous scaffolds with a GFP gene or GFP gene/ branched $25 \mathrm{kD}$ PEI complex at 0 and $10 \mathrm{kV}$ (Fig. $7 \mathrm{a}$ and b). As a result, cells were not only delivered onto nanofibrous scaffolds, but also transfected in the $10 \mathrm{kV}$ group (Fig. 7c). Our study suggests a new application for BES. NVG-BES not only can deliver cells to any substrate, such as patches or scaffolds, but also transfers genes to cells in the non-adhesive condition at the same time. Furthermore, the method can be used to prepare and deliver induced pluripotent stem cells (iPSCs) from fibroblasts with Yamanaka factors. If it can turn fibroblasts extracted from patients directly into stem cells, a novel treatment technique in stem cell therapy could be presented. However, NVGBES with PEI is cytotoxic to cells. Thus, we will improve the BES method by applying a less toxic cationic-polymer than 25 kD PEI. Although it is hard to control BES conditions, this technique can be used as a new transfection method and applied in tissue engineering.

\section{Conclusions}

In summary, we developed a novel NVG-BES system that can simultaneously deliver cells and induce gene transfection. First, we confirmed the voltages suitable for cell viability after BES. Voltages below $10 \mathrm{kV}$ were appropriate for BES due to the resultant good cell viabilities. Second, the cell viability of BES with PEI decreased significantly in comparison with the groups with BES without PEI. Third, we discovered that PBS is the best solution for BES and BES at $10 \mathrm{kV}$ has the highest gene transfection efficiency. Finally, it was possible to directly seed transfected living cells on scaffolds using the BES technique.

\section{Materials and methods}

\section{Cell preparation}

NIH-3T3 (ATCC, USA) cells were cultured in a T75 flask (NUNC) for 2 days with Dulbecco's Modified Eagle's Medium (DMEM, Welgene Inc., Republic of Korea) containing antibiotics (Welgene Inc., Republic of Korea) and 10\% foetal bovine serum (FBS, HyClone, USA). NIH-3T3 cells were seeded at a density of $2 \times$ $10^{6}$ cells per $\mathrm{mL}$. After 2 days, the cells were harvested.

\section{Bio-electrospray}

The non-viral gene delivery-based bio-electrospray (NVG-BES) system was set on a clean bench. The NVG-BES system consisted of two main components: syringe pump (KDS100, 789100, kdScientific, USA) and high voltage generator (DC(+) TKM45K5M, TEKKAM, Republic of Korea). The solution of harvested cells with culture medium was placed in a $10 \mathrm{~mL}$ syringe. Then, the syringe was fixed on a syringe pump. Next, a new $35 \mathrm{~mm}$ dish (NUNC, USA) was filled with culture medium and placed on a support. The cell suspension in the syringe was then electrosprayed using a syringe pump and a high voltage supply at various voltages between the syringe tip and a grounded clip on the $35 \mathrm{~mm}$ dish. The cell suspension was electrosprayed through a needle equipped to the syringe at a flow rate of $10 \mathrm{~mL} \mathrm{~h}^{-1}$. The electrosprayed cells were dropped on a target $35 \mathrm{~mm}$ dish filled with culture medium. The distance between the syringe tip and the grounded clip was $3 \mathrm{~cm}$. Electrospray was performed at room temperature.

\section{Observation of cell morphology}

One day after bio-electrospray, the cell morphology was observed using a microscope (Ti-E, Nikon, Japan). The cell density for this BES experiment was $5 \times 10^{5} \mathrm{~mL}^{-1}$ and the voltage of the electrospray was varied $(0 \mathrm{kV}, 10 \mathrm{kV}, 15 \mathrm{kV}$, and $20 \mathrm{kV})$.

\section{Live/dead assay}

To investigate cell viability after BES with PEI/DNA, a Live/Dead Cell Assay kit (Abcam ab115347, Mitosciences, USA) was used after 1 day of bio-electrospray with a branched $25 \mathrm{kD}$ PEI/DNA complex. When bio-electrospray was run on the dish, the density of cells was $5 \times 10^{5} \mathrm{~mL}^{-1}$ and the electrospray voltage was varied $(0 \mathrm{kV}, 10 \mathrm{kV}, 15 \mathrm{kV}$, and $20 \mathrm{kV})$. The concentration of provided live/dead dye was $1000 \times$. When dye was used for the assay, it had to be diluted $5 \times$ in PBS. The cells were stained with $5 \times$ live/dead dye and incubated 20 minutes at room temperature. Then, the dyed cells were observed by fluorescence microscopy (Ti-E, Nikon, Japan).

\section{Cell viability assay after BES}

To measure cell viability quantitatively, a WST assay was carried out after bio-electrospray. The cell density was $5 \times 10^{5} \mathrm{~mL}^{-1}$ and the electrospray voltage was varied $(0 \mathrm{kV}, 10 \mathrm{kV}, 15 \mathrm{kV}$, $20 \mathrm{kV})$. One day after electrospray, cell viability assay reagent (EZ-cytox, EZ-3000, DOGEN, Republic of Korea) was added to each cell culture dish and the culture medium in the dish and the reagent were allowed to react for $3 \mathrm{~h}$. Then, each sample was quantified using a Sunrise TM absorbance reader (TECAN, Switzerland) at $450 \mathrm{~nm}$. In addition groups with voltage up to $10 \mathrm{kV}(0 \mathrm{kV}, 2.5 \mathrm{kV}, 5 \mathrm{kV}, 7.5 \mathrm{kV}$, and $10 \mathrm{kV})$ were run by the same method to verify the results.

\section{Transfection by NVG-BES}

Transfection by bio-electrospray was performed using several mixing solutions. NIH-3T3 cells were cultured in a T75 flask (NUNC) for 2 days with DMEM containing antibiotics and $10 \%$ FBS. NIH-3T3 cells were seeded at a density of $2 \times 10^{6}$ cells per $\mathrm{mL}$. After 2 days, various BES mixing solutions were tested for transfection efficiency when the cells were harvested and electrosprayed: phosphate buffer saline (PBS, Welgene Inc., Korea), deionized water (DW), DMEM, and DMEM with serum. The cells with various mixing solutions $(500 \mu \mathrm{L})$ and branched PEI25kD/DNA polyplexes $(200 \mu \mathrm{L})$ were mixed and placed in 
a $10 \mathrm{~mL}$ syringe. The branched PEI25kD/DNA polyplexes were used at a $15 \mathrm{~N} / \mathrm{P}$ ratio. The $25 \mathrm{kDa}$ branched PEI was purchased from Sigma Aldrich (408727-100ML, Sigma, USA). The solution was electrosprayed using a syringe pump and a high voltage supply at $10 \mathrm{kV}$ between a syringe tip and a grounded clip on a $35 \mathrm{~mm}$ dish filled with culture medium. The amount of cells electrosprayed was $5 \times 10^{5} \mathrm{~mL}^{-1}$. As soon as electrospraying was complete, fresh culture medium $(1 \mathrm{~mL})$ was added to the dish.

\section{Cell viability assay after NVG-BES}

NIH-3T3 cells were seeded at a density of $2 \times 10^{6}$ cells per mL. After 2 days, the cells were harvested and electrosprayed with only DNA or a DNA-PEI complex in PBS at several voltages. The harvested cells with PBS $(500 \mu \mathrm{L})$ and branched PEI25kD/DNA polyplexes $(200 \mu \mathrm{L})$ or only DNA solution $(200 \mu \mathrm{L})$ were mixed and placed in a $10 \mathrm{~mL}$ syringe. The branched PEI25kD/DNA polyplexes were used at a $15 \mathrm{~N} / \mathrm{P}$ ratio. The cell suspension with only DNA or DNA/PEI complex was electrosprayed using a syringe pump and a high-power supply at various voltages $(0 \mathrm{kV}, 5 \mathrm{kV}, 10 \mathrm{kV}$, and $15 \mathrm{kV}$ ) between the syringe tip and a grounded clip on a $35 \mathrm{~mm}$ dish filled with culture medium. The amount of cells electrosprayed was $5 \times 10^{5} \mathrm{~mL}^{-1}$. As soon as the electrospraying was complete, fresh culture medium $(1 \mathrm{~mL})$ was added to the dish. After 1 day, the cell morphology was observed by microscope (Ti-E, Nikon, Japan) and cell viability was measured using a WST-1 assay kit (EZ-cytox, EZ3000, DOGEN, Republic of Korea).

\section{Transfection at different voltages of NVG-BES}

NIH-3T3 cells were cultured in a T75 flask (NUNC) for 2 days with DMEM containing antibiotics and 10\% FBS. NIH-3T3 cells were seeded at a density of $2 \times 10^{6}$ cells per mL. After 2 days, various electrospray voltages were tested for transfection efficiency. Phosphate buffer saline (PBS, Welgene Inc., Republic of Korea) was used as the BES solution. The solutions of harvested cells in PBS $(500 \mu \mathrm{L})$ and PEI25k/DNA polyplexes $(200 \mu \mathrm{L})$ were mixed and placed in a $10 \mathrm{~mL}$ syringe. The solution was electrosprayed using a syringe pump and a high-power supply at several voltages $(0 \mathrm{kV}, 5 \mathrm{kV}, 10 \mathrm{kV}$, and $15 \mathrm{kV})$ between the syringe tip and a grounded clip on a $35 \mathrm{~mm}$ dish filled with culture medium. The cell density of the electrosprayed cells was $5 \times 10^{5} \mathrm{~mL}^{-1}$. As soon as electrospray was complete, fresh culture medium $(1 \mathrm{~mL})$ was added to the dish.

\section{The fabrication of PCL nanofiber scaffold}

The PCL nanofiber scaffold was fabricated using poly( $\varepsilon$-caprolactone) (PCL, MW: 80 000, Sigma Aldrich, USA) and the electrospinning technique. To prepare the polymer solution for electrospinning, PCL was dissolved in 2,2,2-trifluoroethanol (TFE) (ReagentPlus ${ }^{\circledR} \geq 99 \%, M_{\mathrm{w}}=100.04 \mathrm{~g} \mathrm{~mol}^{-1}$, Sigma Aldrich, USA) at 16\% weight/volume and acetic acid (F.W. 60.05, Duksan, Republic of Korea) was added at $2 \% \mathrm{v} / \mathrm{v}$. Fiber was collected at $18 \mathrm{kV}\left(1.8 \mathrm{kV} \mathrm{cm}^{-1}\right)$ and syringe pump rate of $0.6 \mathrm{~mL} \mathrm{~h} \mathrm{~h}^{-1}$ for $30 \mathrm{~min}$. The electrospinning scaffold was attached to the PDMS layer. Then, $20 \mathrm{~mm}$ diameter samples were obtained by punching, washed with $70 \% \mathrm{EtOH}$, and then washed with DPBS three times before BES. Then, the nanofiber scaffold samples were attached on $35 \mathrm{~mm}$ dishes.

\section{The delivery of transfected living cells by NVG-BES}

NIH-3T3 cells were cultured in a T75 flask (NUNC) for 2 days with DMEM containing antibiotics and 10\% FBS. NIH-3T3 cells were seeded at a density of $2 \times 10^{6}$ cells per mL. After 2 days, BES was applied on a PCL nanofiber scaffold. Phosphate buffer saline (PBS, Welgene Inc., Republic of Korea) was used as the BES solution. The solutions of harvested cells in PBS $(500 \mu \mathrm{L})$ and PEI25k/DNA polyplexes $(200 \mu \mathrm{L})$ were mixed and placed in a $10 \mathrm{~mL}$ syringe. The solution was electrosprayed using a syringe pump and a high-power supply at $0 \mathrm{kV}$ and $10 \mathrm{kV}$ between the syringe tip and a grounded clip on a $35 \mathrm{~mm}$ dish containing PCL scaffold filled with culture medium. The cell density of the electrosprayed cells was $5 \times 10^{5} \mathrm{~mL}^{-1}$. As soon as electrospray was complete, fresh culture medium $(1 \mathrm{~mL})$ was added to the dish.

\section{The turbo green fluorescent protein (tGFP) assay}

The green fluorescent protein (GFP) gene was obtained from Clontech (Palo Alto, CA, USA). PEI25K/GFP (4 $\mu \mathrm{g})$ polyplexes were used at a $15 \mathrm{~N} / \mathrm{P}$ ratio. After transfection by bioelectrospray using PEI/GFP, cells were cultured for $48 \mathrm{~h}$ in a $\mathrm{CO}_{2}$ incubator. The transfected cells were then observed by fluorescence microscopy (Ti-E, Nikon, Japan).

\section{Luciferase assay}

Luciferase reporter, pGL3-vector with SV-40 promoter, and enhancer encoding firefly (Photinus pyralis) luciferase were obtained from Promega (Madison, WI, USA). PEI25K/pGL3 $(4 \mu \mathrm{g})$ polyplexes were used at a $15 \mathrm{~N} / \mathrm{P}$ ratio. After transfection by bioelectrospray using PEI25K/pGL3, cells were cultured for $48 \mathrm{~h}$ in a $\mathrm{CO}_{2}$ incubator. The luciferase assay was then performed according to the manufacturer's protocol. A multiple plate reader (Victor3, Perkin Elmer, USA) was used to measure relative light units (RLUs) (normalized by protein concentration) in the cell extract estimated using a BCA protein assay kit (Pierce Biotechnology, Rockford, IL, USA).

\section{Statistical data analysis}

The statistical analysis was performed using R v3.2.1 software (The R Foundation, http://www.r-project.org). The least significant difference (LSD) method, Duncan's test, and one-way ANOVA were used to compare the means of the properties of the samples. The level of significance was $p<0.05$. The data were reported as the mean \pm standard deviation, $n=3$.

\section{Conflicts of interest}

The authors declare no conflict of interest. 


\section{Acknowledgements}

This study was supported by Basic Science Research Program through the National Research Foundation of Korea (NRF) funded by the Ministry of Science, ICT \& Future Planning (2017R1C1B2011672). This work was supported by Korea Institute of Planning and Evaluation for Technology in Food, Agriculture, Forestry (IPET) through Agri-Bio industry Technology Development Program funded by Ministry of Agriculture, Food and Rural Affairs (MAFRA) (116135-3).

\section{Notes and references}

1 T. K. Kim and J. H. Eberwine, Anal. Bioanal. Chem., 2010, 397, 3173-3178.

2 W. S. Pear, G. P. Nolan, M. L. Scott and D. Baltimore, Proc. Natl. Acad. Sci. U. S. A., 1993, 90, 8392-8396.

3 D. Fischer, T. Bieber, Y. Li, H. P. Elsasser and T. Kissel, Pharm. Res., 1999, 16, 1273-1279.

4 K. Kunath, J. Controlled Release, 2003, 89, 113-125.

5 S. Mehier-Humbert and R. H. Guy, Adv. Drug Delivery Rev., 2005, 57, 733-753.

6 Y. Okubo, K. Ikemoto, K. Koike, C. Tsutsui, I. Sakata, O. Takei, A. Adachi and T. Sakai, Angew. Chem., 2008, 47, 1429-1431.

7 K. Ikemoto, I. Sakata and T. Sakai, Sci. Rep., 2012, 2, 289.

8 S. Sahoo, W. C. Lee, J. C. Goh and S. L. Toh, Biotechnol. Bioeng., 2010, 106, 690-698.
9 R. P. Hall, C. M. Ogilvie, E. Aarons and S. N. Jayasinghe, Analyst, 2008, 133, 1347-1351.

10 E. Ward, E. Chan, K. Gustafsson and S. N. Jayasinghe, Analyst, 2010, 135, 1042-1049.

11 S. C. De Smedt, J. Demeester and W. E. Hennink, Pharm. Res., 2000, 17, 113-126.

12 B. Lee, H. Chae, T. N. Tuyen, D. Kang, H. Kim, M. Lee and S. Ihm, Int. J. Mol. Med., 2009, 23, 759-762.

13 A. V. Kabanov, Pharm. Sci. Technol. Today, 1999, 2, 365-372.

14 G. Nchinda, K. Uberla and O. Zschornig, BMC Biotechnol., 2002, 2, 12.

15 J. H. S. Kuo, Biotechnol. Appl. Biochem., 2003, 37, 267-271.

16 S. Chesnoy and L. Huang, Mol. Ther., 2002, 5, 57-62.

17 A. K. Roos, F. Eriksson, D. C. Walters, P. Pisa and A. D. King, Mol. Ther., 2009, 17, 1637-1642.

18 J. Kang, S. Ramu, S. Lee, B. Aguilar, S. K. Ganesan, J. Yoo, V. K. Kalra, C. J. Koh and Y. K. Hong, Anal. Biochem., 2009, 386, 251-255.

19 D. C. Chang and T. S. Reese, Biophys. J., 1990, 58, 1-12.

20 E. Neumann, M. Schaeferridder, Y. Wang and P. H. Hofschneider, EMBO J., 1982, 1, 841-845.

21 J. Gehl, Acta Physiol. Scand., 2003, 177, 437-447.

22 J. M. Deitzel, J. D. Kleinmeyer, J. K. Hirvonen and N. C. B. Tan, Polymer, 2001, 42, 8163-8170.

23 Y. S. Zheng, R. H. Gong and Y. C. Zeng, RSC Adv., 2015, 5, 48533-48540. 